\title{
Efectividad de la docencia y actitud hacia la lectura en estudiantes de educación primaria
}

\author{
Effectiveness of teaching and attitude towards reading in \\ primary school students
}

Céspedes Arosemena María Isabel

ysaces@hotmail.com

Universidad Nacional de Educación Enrique Guzmán y Valle

\section{Resumen:}

e presenta un estudio cuyo objetivo fue determinar la relación existente entre efectividad de la docencia y actitud hacia la lectura en estudiantes de $5^{\circ}$ y $6^{\circ}$ grado educación primaria. El diseño de investigación fue el correlacional, se utilizaron como instrumento de evaluación la escala de Opinión de los Estudiantes sobre la Efectividad de la Docencia (EOEED) en Educación Superior, elaborado por Rocha (2013), Escala de 17 ítems para recabar la valoración de los alumnos sobre el docente y el cuestionario de Actitudes hacia la Lectura elaborado por Cubas (2007), cada afirmación tiene dos alternativas de respuesta: "De acuerdo" y "En desacuerdo". La muestra estuvo constituido por 80 estudiantes de educación primaria de la Institución Educativa No 1522 Miguel Grau Pucala-Lambayeque. El análisis de los datos permiten concluir que existe una relación estadísticamente significativa entre efectividad de la docencia y actitud hacia la lectura en estudiantes de $5^{\circ}$ y $6^{\circ}$ grado educación primaria

\section{Summary:}

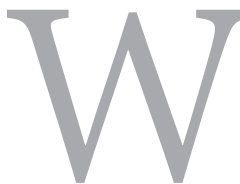

'e present a study whose objective was to determine the relationship between teaching effectiveness and attitude toward reading in students in 5th and 6th grade primary education. The research design was the correlational, the instrument used was the scale of Student Opinion on Teaching Effectiveness (EOEED) in Higher Education, elaborated by Rocha (2013), was used as an instrument of evaluation, the scale has 17 items to collect the assessment of the students on the teacher and the attitudes toward reading questionnaire developed by Cubas (2007), each statement has two alternative answers: "Agree" and "Disagree". The sample was constituted by 80 students of primary education of the Institution Educative $\mathrm{N}^{\circ}$ 1522 Miguel Grau Pucala-Lambayeque. The analysis of the data allows us to conclude that there is a statistically significant relationship between teaching effectiveness and attitude towards reading in students in grades 5 and 6 
Palabras clave: efectividad docente, actitud hacia el estudio, personalidad del docente, características de evaluación, didáctica de enseñanza
Key words: teacher effectiveness, attitude toward the study, teacher personality, evaluation characteristics, teaching didactics

\section{Introducción}

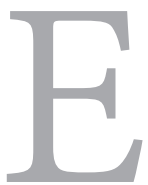

1 propósito de este estudio es analizar la relación existente entre la efectividad docente y actitud frente a la lectura en una muestra deestudiantes de educación primaria de la ciudad de Chiclayo-Perú. Según Medley y Shannon (1994) toda evaluación en contexto de los maestros debe basarse en datos acerca de la efectividad docente, sin embargo, debido a la deficiencia sobre información directa a cerca del tema, muchas evaluaciones de docentes están basadas en información sobre la competencia docente o desempeño" (p. 601). El término efectividad docente, hace referencia a un conjunto de características, competencias y conductas de los docentes en todos los niveles educativos que permitan a los estudiantes alcanzar los resultados deseados, que pueden incluir el logro de objetivos de aprendizajes específicos, además de, adquirir habilidades para resolver problemas, pensar críticamente, trabajar colaborativamente y transformarse en instructores efectivos (Hunt, 2004). En palabras de Dunkin (1997), la efectividad docente es una cuestión referida a la capacidad del mismo para lograr los efectos deseados sobre los estudiantes. Además, define la competencia docente como el conocimiento, las habilidades necesarias y del desempeño como la forma en que este se conduce durante el proceso de enseñar. Si bien es cierto que las competencias y habilidades de los docentes están muy bien orientadas, existen condiciones adversas más fuertes que influyen de manera general en potenciar estas condiciones.

En el contexto de la educación peruana, el reto de la educación en contextos de interacción relativa es contar con docentes bien preparados, tanto a nivel de su disciplina de estudio y especialización, como a nivel de competencias pedagógicas necesarias para generar aprendizajes pertinentes. Durante muchotiempolas iniciativas de formación docente repiten, en la mayoría de los casos, una enseñanza tradicional, basada en cursos teóricos que dejan a la suerte de cada profesor, no se articula la teoría con su práctica pedagógica (Rodríguez, 2013) y en este caso, son los estudiantes los que quedan decepcionados porque no aprendieron lo que debería, y un gran porcentaje la efectividad docente a partir de su vocación de servicio, es lo que a estos niños no les ha permitido cumplir con un buen aprendizaje.

Un estudio en un programa de formación inicial de docentes se observó que para muchos, la docencia no había sido la primera elección, sino que optaron por ser la carrera más barata y con admisión más fácil (Oliart, en Arregui et al., 1996, p. 2). Vaillant (2004) señala que los formadores de las instituciones de formación docente en América Latina son considerados de bajo estatus y usualmente no cuentan con una preparación especial para sus funciones. Algunos 


\section{Artículos Empíricos}

no tienen experiencia de aula, porque ocuparon sus cargos apenas culminaron sus estudios en la misma institución.

Tal vez, estas condiciones del profesorado sean una de los factores que afecten al estudiante, en su actitud frente al estudio en cualquier nivel y en cualquier área, debido a que por lo general una actitud es definida como una tendencia a evaluar una entidad con algún grado de aceptación o rechazo, normalmente expresado en respuestas cognitivas, afectivas o conductuales (Eagly y Chakine, 1993; citado por Aiken, 2002). Un docente que no tengan estas competencias cognitivas, afectivas y conductuales es más seguro que no se efectivo a la hora de enfrentar las tareas académicas.

A través de esta investigación, se pretende contribuir a la discusión sobre modelos de formación sostenible y pertinente, para contribuir a la mejora de la docencia escolar, desde una perspectiva innovadora y sobre todo desde una perspectiva empírica. Además se busca una correlación con la actitud que tienen los estudiantes hacia las tareas escolares, específicamente hacia la lectura. Según Thorne y Pinzás (1988), la lectura es la única actividad que constituye, al mismo tiempo, materia de instrucción, aunque en el Perú sólo signifique esto en primer grado de primaria e instrumento para el aprendizaje. Por ello, uno de los múltiples retos que la escuela debe cumplir es hacer que todos sus estudiantes lean correctamente $\mathrm{y}$ descubran a la lectura como un medio para lograr otros aprendizajes (Solé, 2000).

En este sentido, tratamos de investigar si existe una relación entre la efectividad del docente y la actitud frente hacia la lectura en estudiantes de educación primaria. Esta investigación puede ayudarnos a reorientar nuestro enfoque sobre la actitud del estudiante frente a la lectura, debido que en los últimos años nuestro país a estado en los últimos lugares de evaluación en matemática y comprensión de lectura.

En los últimos años se ha destacado la importancia de la evaluación de la efectividad académica para la mejora de la calidad educativa. Por ello, es importante seguir ahondando en el tema con investigaciones empíricas para dar soporte a la teoría y a las esporádicas investigaciones que nos hacen ver que la efectividad docente es muy importante para el desarrollo del estudiante y sobre todo de la calidad educativa.

\section{Método}

\section{Participantes:}

La muestra estuvo constituida por 40 estudiantes de 5 to grado y 40 estudiantes del sexto grado de educación primaria de la Institución Educativa N 1522 Miguel Grau Pucala-Lambayeque.

\section{Materiales:}

Para la presente investigación se utilizaron los siguientes instrumentos: Escala de opinión de los estudiantes sobre la efectividad de la docencia (EOEED) en Educación Superior, elaborado por Rocha (2013) la cual cuenta con17 ítems para recabar la valoración de los estudiantes sobre el docente, integrando cuatro de las dimensiones reportadas en la literatura y asociadas a su efectividad: relación profesor-alumno (3 ítems), personalidad del docente ( 4 ítems), la evaluación del estudiante realizada por el profesor (5 ítems), y su método de enseñanza (5 ítems). 
Y para evaluar las actitudes hacia la lectura se utilizó el cuestionario de Actitudes hacia la lectura elaborado por Cubas (2007), cada afirmación tiene dos alternativas de respuesta: "De acuerdo" y "En desacuerdo". Los ítems de este instrumento son calificados de acuerdo a si son directos (expresan una actitud favorable hacia la lectura), o inversos (expresan una actitud desfavorable hacia la lectura). Los ítems directos obtienen el puntaje de uno si la respuesta es "De acuerdo" y de cero si es "En desacuerdo"; mientras que los ítems inversos obtienen el puntaje de uno si la respuesta es "En desacuerdo" y de cero si es "De acuerdo". El Cuestionario de Actitudes hacia la Lectura (ver Anexo) está conformada por 42 ítems: 22 directos $(1,4,11,12,13,15,19,20,21$, $22,24,25,26,29,30,32,34,35,37,38,39$ y 42) у 20 inversos $(2,3,5,6,7,8,9,10,14,16,17,18$, $23,27,28,31,33,36,40$ y 41$)$.

Según el análisis de confiabilidad, se encontró un Alfa de Cronbach de .886, para la Escala de opinión de los estudiantes sobre la efectividad de la docencia (EOEED) en Educación Superior, para la dimensión relación profesor alumno el puntaje de Alfa de Cronbach fue de 0.714; personalidad del profesor .677; evaluación .385 y aspectos didácticos .918. Y el alfa de Crombach del cuestionario de actitudes hacia la lectura presentó una alta consistencia interna con un puntaje de 0.784 de Alfa.

\section{Resultados}

Los resultados se analizaron en dos niveles; desde el nivel descriptivo presentados en la tabla 1 evidencian que desde la percepción de los estudiantes siempre los docentes tienen buena efectividad y que además tienen muy buena relación con sus profesores. Por otro lado está referido también que tienen un nivel alto, en la tabla 2 , de comprension de lectura. Con ello evidenciamos que el nivel de aprendizaje podría estar bastante alto o al menos el docente no es el problema.

Tabla 1

Frecuencia de la variable efectividad de la docencia

\begin{tabular}{lcccc}
\hline & Frecuencia & Porcentaje & Porcentaje válido & $\begin{array}{c}\text { Porcentaje } \\
\text { acumulado }\end{array}$ \\
\hline A veces & 2 & 2.5 & 2.5 & 2.5 \\
Siempre & 78 & 97.5 & 97.5 & 100 \\
Total & 80 & 100 & 100 & \\
\hline
\end{tabular}


Tabla 2

Frecuencia de la variable actitud hacia la lectura

\begin{tabular}{lcccc}
\hline & Frecuencia & Porcentaje & Porcentaje válido & $\begin{array}{c}\text { Porcentaje } \\
\text { acumulado }\end{array}$ \\
\hline De acuerdo & 73 & 91.25 & 91.25 & 91.25 \\
En desacuerdo & 7 & 8.75 & 8.75 & 100 \\
Total & 80 & 100 & 100 & \\
\hline
\end{tabular}

En cuanto al nivel inferencial en la tabla 3, se evidencia el análisis de relación entre las dos variables mediante el Coeficiente de correlación de Pearson (r), posteriormente en la Tabla 4 se realiza la relación entre las dimensiones de la variable efectividad de la docencia y la variable actitud hacia la lectura en estudiantes de 5 to grado y 40 estudiantes del sexto grado de educación primaria de la Institución Educativa No 1522 Miguel Grau Pucala-Lambayeque.

Tabla 3

Relación entre efectividad de la docencia y actitudes hacia la lectura

\begin{tabular}{llll}
\hline & & Efectividad & Actitud \\
\hline Efectividad & Correlación de Pearson & 1 & $.498\left(^{* *}\right)$ \\
& Sig. (bilateral) & & .000 \\
Actitud & Correlación de Pearson & $.498\left(^{* *}\right)$ & 1 \\
& Sig. (bilateral) & .000 & \\
& $\mathrm{~N}$ & 80 & 80 \\
\hline
\end{tabular}

** La correlación es significativa al nivel 0,01 (bilateral).

De acuerdoala tabla mostrada, el coeficientede correlación de Pearson $r=0,498$, en consecuencia existe una relación estadísticamente significativa entre efectividad de la docencia y actitud hacia la lectura en estudiantes de $5^{\circ}$ y $6^{\circ}$ grado educación primaria de la Institución Educativa $\mathrm{N}^{\circ} 1522$ Miguel Grau Pucala-Lambayeque, según el cuadro de índices de correlación propuesto por Hernández, Fernández y Baptista (2010) es correlación positiva media. 
Tabla 4

Correlación entre las dimensiones de efectividad docente y actitud hacia la lectura

\begin{tabular}{lll}
\hline & & Actitud \\
\hline Efectividad & Correlación de Pearson & $.498\left(^{* *}\right)$ \\
& Sig. (bilateral) & .000 \\
Relación profesor alumno & Correlación de Pearson & $.374\left(^{* *}\right)$ \\
& Sig. (bilateral) & .001 \\
Personalidad & Correlación de Pearson & $.437\left(^{* *}\right)$ \\
Evaluación & Sig. (bilateral) & .000 \\
& Correlación de Pearson & $.562\left(^{* *}\right)$ \\
Aspectos didácticos & Sig. (bilateral) & .000 \\
& Correlación de Pearson & $.325\left(^{* *}\right)$ \\
& Sig. (bilateral) & .003 \\
\hline
\end{tabular}

** La correlación es significativa al nivel 0,01 (bilateral).

De acuerdo a la tabla mostrada, el coeficiente de correlación de Pearson para las relaciones entre las dimensiones de la variable efectividad de la docencia y actitudes hacia la lectura es en la mayor parte una relación positiva media y baja, en consecuencia existe una relación estadísticamente significativa entre las dimensiones de efectividad de la docencia y actitud hacia la lectura en estudiantes de $5^{\circ}$ y $6^{\circ}$ grado educación primaria de la Institución Educativa $\mathrm{N}^{\circ} 1522$ Miguel Grau Pucala-Lambayeque, según el cuadro de índices de correlación propuesto por Hernández, Fernández y Baptista (2010) es correlación positiva media y baja.

\section{Discusión}

En este apartado se discuten los resultados a los cuales se ha llegado a partir del análisis estadístico de los resultados. En primer lugar se discutirán los resultados estadísticos a nivel descriptivo y posteriormente los resultados inferenciales.

A nivel descriptivo los resultados reportan que existe un nivel alto de percepción sobre la efectividad de la docencia que presentan los estudiantes de educación primaria. Estos resultadosson acordes con los reportes de Sanzana (2012) quien en una muestra de docentes de chile reportan los mismos resultados al nuestro, en ese sentido, pareciera que la práctica efectiva se refieren a la utilización del tiempo de forma efectiva, organización de la materia, enseñanza de estrategias de aprendizaje, enseñanza directa, uso de organizadores previos, la promoción del aprendizaje colaborativo, el desarrollo de actividades tutoriales y la adaptación de la enseñanza; al respecto los docentes afirman que 
hacen un uso frecuenta de todas estas actividades en el aula, además, mencionan también que el razonamiento pedagógico que conduce a estas acciones se ajusta mayoritariamente al discurso teórico que caracteriza a cada una de ellas. Estas evidencias también están acordes con los reportes de (Marsh, 1984, 1987) quien también menciona que para que haya una buena percepción sobre la efectividad de enseñanza del docente es necesario tomar en cuenta la claridad explicativa, la preparación y organización de las clases, rigor y competencia del profesor, habilidades comunicativas e interacción con el grupo. Por su parte, estudios de Chirinos y Padrón (2010) muestran una eficiencia centrada en la maximización de los recursos y el desarrollo de las potencialidades del estudiante. Para ser mejor docente, según los reportes de Fernándes y Gómez (2006) los docentes utilizan estrategias, en la mayoría de ocasiones, preinstruccionales, coinstruccionales y posinstruccionales.

Existe un nivel bajo de actitud hacia la lectura que presentan los estudiantes de $5^{\circ}$ y $6^{\circ}$ grado de educación primaria. Estos resultados están acordes con los reportes de Sáenz (2012) quien en su investigación sobre la actitud hacia la lectura en estudiantes de primaria encontró que existe una relación positiva y significativa entre las variables Comprensión Lectora y Actitudes hacia la lectura. Se deberían a que en esta muestra la efectividad de la docencia es alto y esta preocupación o acercamiento con el docente es lo que generaría un alto nivel de actitud hacia la lectura, por otro lado, también los padres tendrían una implicación significativa en estos resultados, en investigaciones previas se ha evidenciado que la falta de apoyo de los padres, poca formación de los maestros, deficiencias del proceso enseñanza-aprendizaje, entre otras (Thorne, 2005; Thorne y Nakano, 2001; entre otros.), por lo general son causas de la falta de actitud hacia la lectura de los estudiantes.

A nivelinferencial, los resultados muestran que existe una relación estadísticamente significativa entre efectividad de la docencia y actitud hacia la lectura en estudiantes de educación primaria. Estos resultados se explicarían en la medida que los estudiantes cuentan con un conjunto de características que hacen que perciban a sus docentes como un buen docente. En cuanto a las características deseables, profesores y estudiantes destacan la preparación y claridad en la impartición de las clases y la adaptación al ritmo de aprendizaje de los estudiantes. Sin embargo, mientras que los estudiantes apuntan a las habilidades comunicativas del profesor, los profesores inciden en la importancia de formar con espíritu crítico y autonomía en el aprendizaje de os estudiantes (Feldman, 1988).

En consecuencia podemos afirmar a partir de estos resultados que dentro de estas habilidades que se mejoran a partir de la efectividad del docente, es la actitud que muestra el estudiante frente a la lectura y al estudio en general. Las actitudes y creencias que un estudiante se va forjando en relación con varios temas en particular pueden ser a la buena relación que tiene con su maestro y si esta relación o efectividad de enseñanza del docente es mala, puede afectar a su forma de vivir en la escuela y por supuesto a la forma de comprender cuando realiza un aprendizaje comprenderlos. Las actitudes de un estudiante hacia la lectura pueden influir en su comprensión del texto. Puede que el estudiante en una actitud negativa posea las habilidades 
requeridas para comprender con éxito un texto, pero su actitud general habrá de interferir con el uso que haga de tales habilidades (Sáenz, 2012).

Dentro de este nivel también, existe una relación estadísticamente significativa entre los factores de la efectividad de la docencia, relaciones profesor - alumnos, personalidad del profesor como docente de grado, evaluación que implementa el profesor de grado, aspectos didácticos de la enseñanza y actitud hacia la lectura en estudiantes de $5^{\circ}$ y $6^{\circ}$ grado educación primaria de la Institución Educativa $\mathrm{N}^{\circ} 1522$ Miguel Grau Pucala-Lambayeque. Estos resultados son explicados desde la perspectiva psicológica de la educación. En este sentido, los pensamientos el profesor son importantes, a sí Murillo (2008) enfatiza que los estudiantes aprenderán en la medida en que el docente confíe en lo que pueden hacer sus estudiantes. Así, de nuevo, las altas expectativas del docente por sus estudiantes se constituyen como uno de los factores de eficacia escolar más determinantes del logro escolar. Pero confiar en los alumnos no es suficiente si éstos no lo saben. De esta forma, elementos ya mencionados tales como la evaluación y, sobre todo, la comunicación frecuente de los resultados, una atención personal por parte del docente o un clima de afecto entre el docente y el estudiantes son factores que contribuyen a que esas altas expectativas se conviertan en autoestima por parte de estos últimos y, con ello, en alto rendimiento.

Estas actitudes de los profesores, harán que los estudiantes también mejoren específicamente en el tema de la lectura, investigaciones previas, Cueto et al. (2003) y Smith (1990) mencionan que la lectura puede mantenerse estables a través de los años, o incluso aumentar. Con estos datos, no resulta extraño encontrar que, el nivel de actitud hacia la lectura de los estudiantes participantes en este estudio, sean positivas y que, con el paso del tiempo, se vayan haciendo más favorables. Esto podría estar indicando que los estudiantes sí conocen y entienden la importancia de la lectura en sus vidas. De igual manera en otro estudio realizado por Lulaico (2010) relacionado a los padres de familia del cuarto grado de primaria, sostiene que los niños aprenden de los hábitos de sus padres. Es decir, que leer en el hogar es muy importante porque el gusto por la lectura comienza en casa, cuando padres e hijos comparten momentos divertidos alrededor de un libro, leen, se cuentan cuentos y conversan. En este sentido, es importante seguir profundizando en el estudio de esta variable y desde diferentes perspectivas.

\section{Conclusiones}

Los resultados obtenidos muestran que la efectividad docente con unas directrices bien definidas, contribuye a una percepción positiva sobre la mejora de la lectura de los estudiantes, por ende tiende a mejorar el rendimiento académico de sus estudiantes a partir de una buena planificación de sus clases y una mejor interacción con los mismos. Asimismo, nuestro estudio nos ha hecho descubrir carencias, sobre todo en relación con los niveles de profundidad de la reflexión realizada por los profesores. Y en este mismo sentido, es el apoyo de los padres que no se evidencia en este estudio, pero que por evidencias anteriores los padres tienen un rol importante en este aprendizaje de los estudiantes. 


\section{Referencias}

Aiken, L. (2002). Attitude and Related

Psychosocial Constructs. Thousand Oaks, EE.UU: Sage Publications.

Arregui, P., Díaz, H. y Hunt, B. (1996). Problemas, Perspectivas y Requerimientos de la Formación Magisterial en el Perú; Informe final del diagnóstico elaborado a solicitud del Ministerio de Educación y la GTZ. Lima, Perú: Grupo de Análisis para el Desarrollo (GRADE).

Chirinos, N. y Padrón, E. (2010). La eficiencia docente en la práctica educativa. Revista de ciencias sociales (RCS) 16(3), 481-492. Recuperado de http://www.redalyc.org/ pdf/280/28016320009.pdf.

Cubas, A. (2007). Actitudes hacia la lectura $y$ niveles de comprensión lectora en estudiantes de sexto grado de primaria (Tesis de Licenciatura). Pontificia Universidad católica del Perú. Recuperado de http://tesis.pucp.edu.pe/files/PUCP.

Cueto, S., Andrade, F. y León, J. (2002). Las Actitudes de los Estudiantes Peruanos hacia la Lectura, Escritura Matemática y Lenguas Indígenas. Documento de trabajo 44 (GRADE). Lima, Perú. Recuperado de http://www.grade.org.pe/download/pubs/ ddt/ddt44.pdf.

Dunkin, M. (1997). Assessing Teachers' Effectiveness. Issues in Educational Research, 7(1). Recuperado de http:// www.iier.org.au/iier7.dunkin.html.
Feldman, K. A. (1988). Effective College Teaching from the Students 'and Faculty' View: Matched or Mismatched Priorities. Research in Higher Education, 28(4), pp. 291-328.

Fernándes, D. y Gómez, R. (2006). Efectividad de las estrategias de enseñanza utilizadas por el docente de educación inicial en el uso del computador en el aula de preescolar. Universidad Católica Andrés Bello. Caracas, Venezuela.

Hunt, B. C. (2004). La Educación Primaria Peruana: Aun Necesita Mejorarse. En ¿Es Posible Mejorar la Educación Peruana? Lima: GRADE: Grupo de Análisis para el Desarrollo.

Lulaico, M.A. (2010). Actitud hacia la lectura en padres de estudiantes de cuarto de primaria de una institución educativa del callao (Tesis de maestría). Universidad San Ignacio de Loyola, lima, Perú.

Marsh, H. W. (1984). Students'evaluations of University Teaching: Dimensionality, Reliability, Validity, Pontial Biases, and Utility. Journal of Educational Psychology, 76, pp. 707-54.

Marsh, H. W. (1987). Students'evaluation of University Teaching: Research Findings, Methodological Issues, and Directions for Future Research. International Journal of Educational Research, 11(3), 253-388. 
Medley, D. M. y Shannon, D. M. (1994). Teacher Evaluation. In T. Husen \& T. N. Postlethwaite (Eds.). The International Encyclopedia of Education (2 ${ }^{\circ}$ ed.) (Vol. 10, 6015-6020). New York: Pergamon.

Murillo, J. (2008). Enfoque, situación y desafíos de la investigación sobre eficacia escolar en América latina y el caribe. En Blanco et al (eds.). Eficacia escolar y factores asociados en América latina y el caribe.

Oficina Regional de Educación de la UNESCO para América Latina y el Caribe. Santiago: Salesianos S.A.

Rocha, R. (2013). Escala de opinión de los estudiantes sobre la efectividad de la docencia (EOEED) en educación superior. Revista formación Universitaria, 6(6), 13-22. doi:10.4067/S071850062013000600003 .

Rodriguez, R. (2013). El desarrollo de la práctica reflexiva sobre el quehacer docente, apoyada en el uso de un portafolio digital, en el marco de un programa de formación para académicos de la Universidad Centroamericana de Nicaragua (tesis de doctorado). Facultad de pedagogía. Universitat de Barcelona. Recuperado de http://repositorio.uca.edu.ni/1754/1/ RRODRIGUES_TESIS\%20Dra..pdf
Sáenz, J.L. (2012). Comprensión lectora y actitudes hacia la lectura en escolares del quinto grado de primaria del asentamiento humano Angamos-Ventanilla (Tesis de maestría). Universidad San Ignacio de Loyola, Lima, Perú.

Sanzana, G. (2012). La práctica de aula: percepción de efectividad u autoeficacia. (Tesis doctoral). Universidad de Córdova. Córdoba, España.

Solé, I. (2000). Estrategias de lectura (11va. ed.). Barcelona: Edit. Grao. Recuperado de http://www.terras.edu.ar/biblioteca/3/Laensenanza-deestrategias.pdf.

Thorne, C. \& Nakano, T. (2001). El rol del maestro de primer grado en el aprendizaje de la lectura en el Perú. En A. Bazán (Eds.). Enseñanza y evaluación de la lectura y escritura: algunos aportes de la investigación y la tecnología (23-40). Sonora: Instituto Tecnológico de Sonora.

Thorne, C. \& Pinzás, J. (1988). Factors affecting reading achievement in Peru. Annual Meeting AERA. New Orleans.

Vaillant, D. (2004). Formación de Docentes en América Latina; Re-inventando el Modelo Tradicional. Barcelona: Octaedro. 


\section{Apéndice}

\section{Escala de Opinión de los Estudiantes sobre la Efectividad de la Docencia (EOEED)}

\begin{tabular}{|c|c|c|c|c|c|}
\hline $\mathbf{N}^{\circ}$ & Ítems & 1 & 2 & 3 & 4 \\
\hline 1 & $\begin{array}{l}\text { El profesor ayuda a superar los problemas relacionados con mi } \\
\text { aprendizaje en esta clase. }\end{array}$ & & & & \\
\hline 2 & $\begin{array}{l}\text { El profesor aclara mis dudas relacionadas con el contenido de este } \\
\text { curso. }\end{array}$ & & & & \\
\hline 3 & $\begin{array}{l}\text { El profesor con frecuencia resuelve los conflictos que tiene con los } \\
\text { alumnos de esta clase. }\end{array}$ & & & & \\
\hline 4 & El profesor de esta clase muestra vocación por enseñar. & & & & \\
\hline 5 & El profesor de esta clase se ha ganado mi respeto & & & & \\
\hline 6 & Respeto al profesor como un docente comprometido. & & & & \\
\hline 7 & En esta clase, me siento escuchado por el profesor. & & & & \\
\hline 8 & $\begin{array}{l}\text { El profesor propicia que supere los errores que cometo relacionados } \\
\text { con el contenido de este curso. }\end{array}$ & & & & \\
\hline 9 & $\begin{array}{l}\text { El profesor suele modificar la clase para mejorar mi aprendizaje del } \\
\text { contenido de este curso. }\end{array}$ & & & & \\
\hline 10 & $\begin{array}{l}\text { El profesor de esta clase asigna calificaciones que reflejan el } \\
\text { dominio de los alumnos en el contenido del curso. }\end{array}$ & & & & \\
\hline 11 & $\begin{array}{l}\text { El profesor de esta clase es justo con los alumnos al asignar } \\
\text { calificaciones. }\end{array}$ & & & & \\
\hline 12 & $\begin{array}{l}\text { El profesor de esta clase asigna calificaciones que reflejan el logro } \\
\text { de cada alumno. }\end{array}$ & & & & \\
\hline 13 & $\begin{array}{l}\text { El profesor toma en cuenta mis conocimientos para propiciar mi } \\
\text { aprendizaje. }\end{array}$ & & & & \\
\hline 14 & El profesor propicia mi participación activa. & & & & \\
\hline 15 & El profesor propicia mi reflexión personal & & & & \\
\hline 16 & $\begin{array}{l}\text { El profesor conduce un repaso cuando detecta que a la mayoría de } \\
\text { los alumnos les falta saber algo para enfrentar un tema con éxito. }\end{array}$ & & & & \\
\hline 17 & $\begin{array}{l}\text { El profesor de este curso organiza los temas en una secuencia } \\
\text { lógica. }\end{array}$ & & & & \\
\hline
\end{tabular}




\section{Cuestionario de Actitudes hacia la Lectura}

A continuación se te presentan una serie de enunciados. Trata de responder a todos ellos con aquello que sientes, piensas o haces. No existen respuestas malas o buenas, sólo es importante que respondas con la mayor sinceridad.

Marca con una X sobre el recuadro que contenga la opción que exprese mejor tu actitud hacia el enunciado.

ítems

1. Me gusta leer en mis ratos libres.

2. Leo solamente en mi colegio. De acuerdo En desacuerdo

3. Sólo me gusta leer los libros que contienen dibujos. De acuerdo En desacuerdo

4. Me gusta leer el periódico. De acuerdo En desacuerdo

5. Cuando estoy de vacaciones no leo. De acuerdo En desacuerdo

6. Creo que no soy bueno para leer. De acuerdo En desacuerdo

7. No me gusta leer en clase. De acuerdo En desacuerdo

8. Me gusta más ver televisión que leer. De acuerdo En desacuerdo

9. Cuando hago mis tareas dejo al final aquéllas en las que tengo que leer. De acuerdo En desacuerdo

10. Leo sólo cuando me obligan. De acuerdo En desacuerdo

11. Me siento feliz cuando me regalan un libro. De acuerdo En desacuerdo

12. Saco voluntariamente libros de la biblioteca del colegio. De acuerdo En desacuerdo

13. Soy bueno en lectura. De acuerdo En desacuerdo

14. Sólo leo cuando alguien me acompaña. De acuerdo En desacuerdo

15. La lectura es importante en mi vida. De acuerdo En desacuerdo

16. Prefiero los videojuegos antes que leer. De acuerdo En desacuerdo

17. Me acerco a un libro por su apariencia. De acuerdo En desacuerdo

18. Me gusta más escuchar música que leer.

19. Leo en los puestos de periódicos. De acuerdo En desacuerdo

20. Me gusta leer en mi casa. De acuerdo En desacuerdo

21. Siempre que salgo a pasear llevo conmigo un libro. De acuerdo En desacuerdo

22. Mis papás piensan que soy un buen lector. De acuerdo En desacuerdo

23. Me da flojera leer. De acuerdo En desacuerdo 


\begin{tabular}{|c|c|}
\hline 24. Si tuviera dinero me compraría libros. De acuerdo En desacuerdo & \\
\hline 25. Cuando puedo voy a bibliotecas o librerías a buscar información. De acuerdo En desacuerdo & \\
\hline 26. Leo cuando estoy en casa. De acuerdo En desacuerdo & \\
\hline 27. Creo que leer no es necesario en mi vida. De acuerdo En desacuerdo & \\
\hline 28. Rechazo los libros "gordos". De acuerdo En desacuerdo & \\
\hline 29. Me gusta leer novelas. De acuerdo En desacuerdo & \\
\hline 30. Leer es fácil. De acuerdo En desacuerdo & \\
\hline 31. Sólo leo cuando lo necesito para hacer mis tareas. De acuerdo En desacuerdo & \\
\hline 32. Mis profesores creen que leo bien. De acuerdo En desacuerdo & \\
\hline 33. Leer es aburrido. De acuerdo En desacuerdo & \\
\hline 34. Me gusta leer revistas. De acuerdo En desacuerdo & \\
\hline 35. Leer novelas es fácil. De acuerdo En desacuerdo & \\
\hline 36. Me pongo nervioso cuando leo. & \\
\hline 37. Me gusta leer historietas. De acuerdo En desacuerdo & \\
\hline 38. Prefiero leer que ver televisión. De acuerdo En desacuerdo & \\
\hline 39. En navidad pido que me regalen un libro. De acuerdo En desacuerdo & \\
\hline 40. Prefiero que me regalen juguetes antes que un libro. De acuerdo En desacuerdo & \\
\hline 41. Las personas que leen son aburridas. De acuerdo En desacuerdo & \\
\hline 42. Cuando leo un libro nunca me siento solo. & \\
\hline
\end{tabular}

